\title{
Evaluation of Cystic and Solid Renal Lesions with Contrast- Enhanced Ultrasound: A Retrospective Study
}

\section{()(1) $\odot \Theta$}

Authors

Arash Najafi ${ }^{1}$, Michael Wildt ${ }^{2}$, Nicolin Hainc ${ }^{3}$, Joachim Hohmann ${ }^{1}$

\begin{abstract}
Affiliations
1 Department of Radiology and Nuclear Medicine, Canton Hospital Winterthur, Winterthur, Switzerland

2 Department of Radiology and Nuclear Medicine, University Hospital Basel, Basel, Switzerland

3 Department of Medical Imaging, Division of Neuroradiology, Toronto Western Hospital, Toronto, Canada
\end{abstract}

Key words

CEUS, Renal, Bosniak, RCC, Contrast-Enhanced Ultrasound

received 16.01 .2021

accepted 15.04.2021

Bibliography

Ultrasound Int Open 2021; 7: E25-E34

DOI 10.1055/a-1522-8969

ISSN 2199-7152

(c) 2021. The Author(s).

This is an open access article published by Thieme under the terms of the Creative Commons Attribution-NonDerivative-NonCommercial-License, permitting copying and reproduction so long as the original work is given appropriate credit. Contents may not be used for commecial purposes, or adapted, remixed, transformed or built upon. (https://creativecommons. org/licenses/by-nc-nd/4.0/)

Georg Thieme Verlag KG, Rüdigerstraße 14,

70469 Stuttgart, Germany

Correspondence

Arash Najafi

Department of Radiology and Nuclear Medicine

Canton Hospital Winterthur

Brauerstrasse 15

8401 Winterthur

Switzerland

Tel.: + 41765054309

najafi.arash@gmail.com

\section{ABSTRACT}

Purpose Renal lesions are frequent random findings on CT, $\mathrm{MRI}$, and conventional ultrasound. Since they are usually found accidentally, the respective examinations have not been performed optimally to provide a conclusive diagnosis, making additional multiphase contrast-enhanced examinations necessary. The aim of the study is to correlate CEUS findings with the final diagnosis and to determine whether it is a suitable method for the conclusive characterization of undetermined renal lesions.

Materials and Methods All CEUS examinations of focal renal lesions performed at our institute between 2007 and 2014 were retrospectively examined. 437 patients with a total of 491 lesions and 543 examinations were included. 54 patients had bilateral lesions. One patient had three lesions in one kidney. Histology was available in 49 cases and follow-ups in 124 cases. The sensitivity, specificity, positive and negative predictive value as well as positive and negative likelihood ratios were calculated.

Results There were 54 malignant and 437 benign lesions. The sensitivity and specificity were $0.981 / 0.954$ overall, $1.000 / 0.956$ for cystic lesions, $0.977 / 0.906$ for solid lesions, and $0.971 / 0.071$ for the histologically confirmed lesions. Bosniak classification was consistent in 289 of 301 lesions (96\%). Only 12 lesions (3.9\%) were falsely assessed as malignant.

Conclusion CEUS is an appropriate method for the clarification of undetermined renal lesions. The characterization of cystic lesions according to Bosniak is adequately possible, especially for potentially malignant lesions (types III and IV).

\section{Purpose}

Cystic renal lesions are common random findings on computed tomography (CT), magnetic resonance imaging (MRI), and conventional native ultrasound (US) [1]. It is assumed that more than half of all patients over 50 years of age have at least one such lesion. They are only clinically significant if they are symptomatic or po-

tentially (pre-)malignant. The CT-based classification of cystic focal kidney lesions was introduced in 1986 by Bosniak et. al. [2] and updated in 2005 [3] and again in 2019 [4]. Solid kidney lesions are much less common but are sometimes found incidentally. For further characterization of both cystic and solid lesions, multiphase contrast-enhanced examinations are necessary [5]. While CT is 
associated with the risks of iodine application and a relatively high radiation exposure [6], MRI is not always readily available and is more expensive. Contrast-enhanced ultrasound (CEUS) is a relatively new method which can display organ perfusion in real time and in recent years has created new options for the assessment of cystic kidney lesions [7]. The available contrast agents consist of gas bubbles and are generally well tolerated, without nephrotoxicity, and rarely cause allergic reactions [8,9]. Studies have shown that CEUS is superior to CT for imaging of vessels in solid lesions and vascularization of septa and walls of complicated cysts [1012]. The reliability of the Bosniak classification for contrast-enhanced ultrasound (CEUS) has been demonstrated in several studies $[13,14]$, whereby ultrasound generally tends to upstage the Bosniak category due to its higher spatial resolution compared to CT. Furthermore, CEUS is helpful in the diagnosis of pseudotumors (e. g., hypertrophic Bertini columns), which exhibit the same contrast agent behavior as the surrounding renal parenchyma in all phases [15-18]. On the other hand, solid lesions usually show different contrast agent enhancement in at least one phase [19].

CEUS has been performed at our institute since 2007 for the further characterization of incidentally found cystic and solid renal lesions. Based on the lesion classification, further diagnostic and/or therapeutic procedures have been determined ( $\vee$ Fig. 1). Lesions are followed up by CEUS if there are not more than two lesions for both kidneys. Otherwise, follow-up is done by means of CT or MRI (if not contraindicated). Cystic and solid lesions suspicious for ma- lignancy are surgically removed in most cases. The aim of the present study is to correlate the findings of CEUS performed in the clinical setting with the final diagnoses and to show whether CEUS is suitable for the characterization of initially unclear kidney lesions.

\section{Materials and Methods}

This single-center retrospective study was conducted according to GCP-ICH guidelines and approved by the regional ethics committee (reference no. EKNZ: 2014-158). All patients who underwent a CEUS examination of the kidneys in the period from January 2007 to January 2014 at our institute were included ( $>$ Fig. 2). In total, there were 463 patients and 572 CEUS examinations. Of these, 14 patients with 15 examinations had to be excluded due to a lack of data. Accordingly, 449 patients and 557 examinations could be included in the study. In 54 of these patients both kidneys were examined. Of the 449 patients, 141 (31.4\%) were female and 308 $(68.6 \%)$ male. At the time of the initial examination, the patients were between 18 and 97 years of age with a median age of 61.6 years (w: 58.8 years; $m$ : 62.8 years). 490 examinations ( $88 \%$ ) were performed during an inpatient stay and 67 (12\%) were performed on an outpatient basis. After the initial assessment, a further 12 patients with 14 examinations, for whom no final clear assessment could be made (mostly due to death or loss of follow-up), had to be excluded. This left 437 patients with 543 CEUS examinations for statistical evaluation. The lesions were classified as solid or cystic,

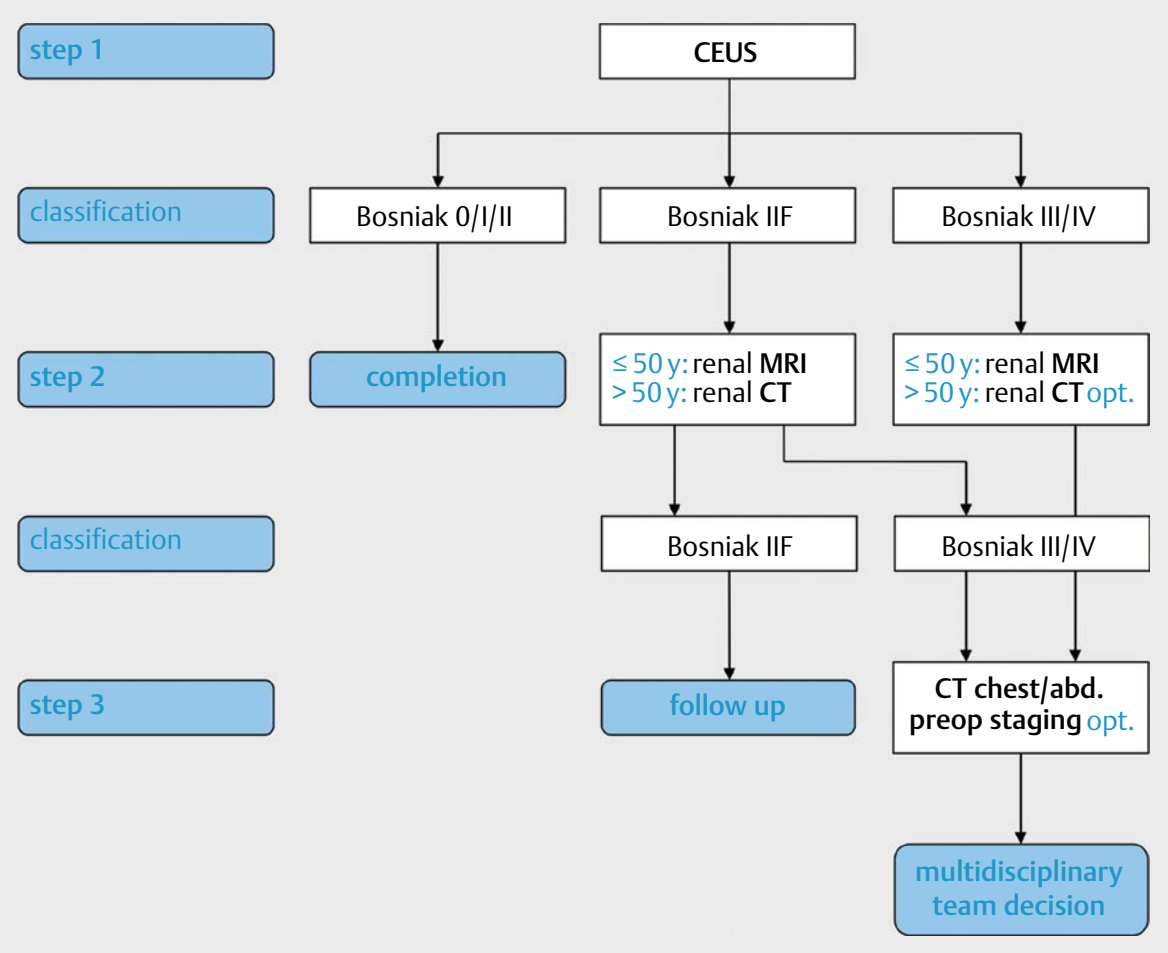

- Fig. 1 Algorithm for the management of cystic kidney lesions. After CEUS and taking into account the initial imaging, the lesion is classified according to Bosniak. In case of > Bosniak II, further imaging with a dedicated CT or MR renal protocol is performed, depending on patient age and possible contraindications. The further procedure depends on the result of all examinations, with the highest classification being decisive. In the algorithm, Bosniak 0 is referred to as a pseudolesion, an inflammatory lesion, or an ischemic lesion. yrs = years, $F U=$ follow-up, $M R I=$ magnetic resonance imaging, CEUS = contrast-enhanced ultrasound , CT = computed tomography.. 


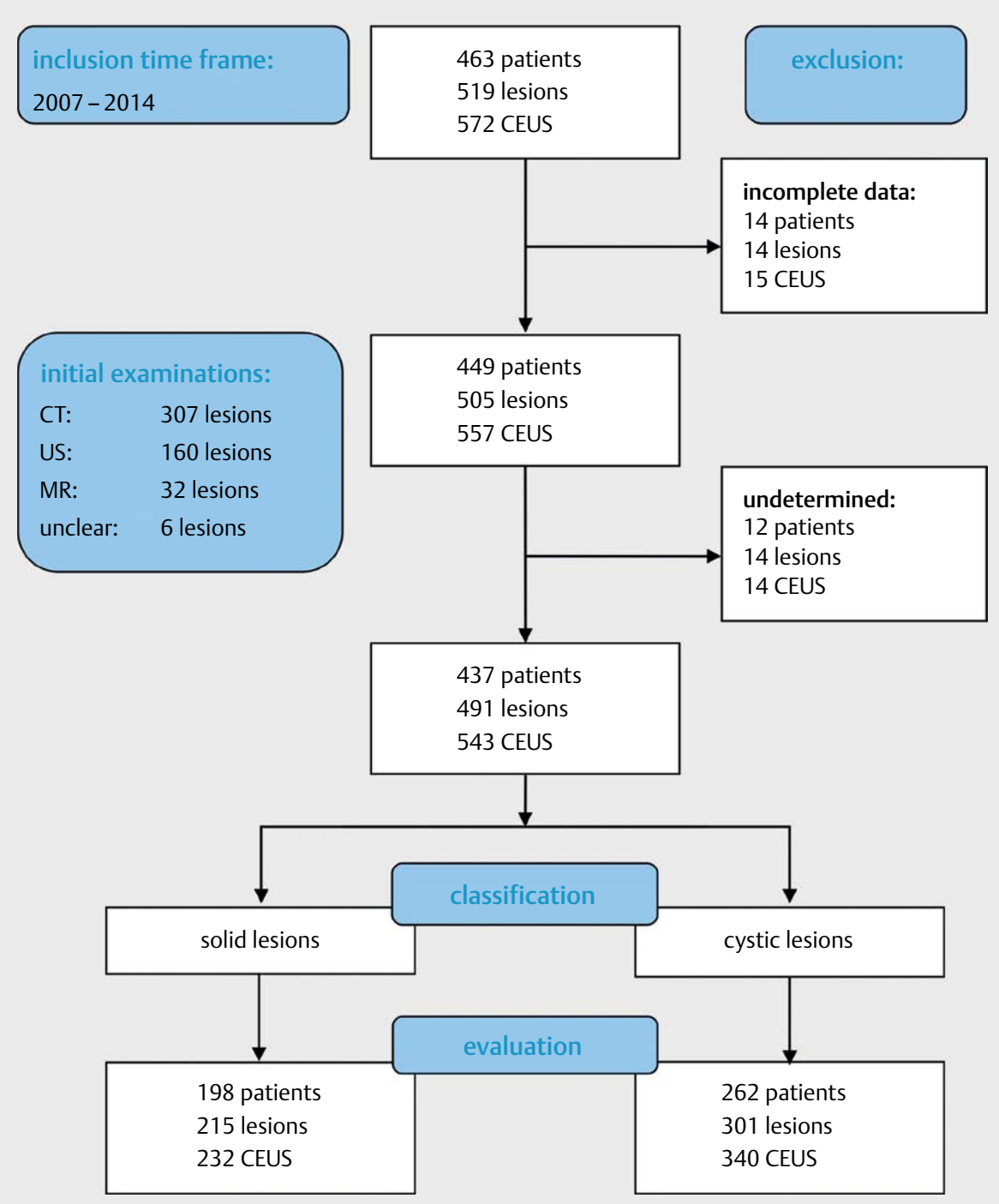

- Fig. 2 Modified CONSORT flowchart for patient recruitment of the study, including number of patients, number of lesion, and CEUS performed (including any follow-ups). The study included all patients who received a renal CEUS examination with an initially unclear lesion on another imaging modality (CT/US/MRI). In six outpatients the initial examination modality could not be determined. Patients with incomplete data $(n=14)$ and those with lesions that remained unclear despite different imaging modalities and/or follow-ups had to be excluded ( $\mathrm{n}=12$ ). The remaining patients were classified according to the morphological lesion criterion into solid and cystic lesions, as is often done clinically. The further introduced subgroup of histologically confirmed lesions is not listed in the flowchart, as it contains both solid and cystic lesions. CONSORT = Consolidated Standards of Reporting Trials, CEUS = contrast-enhanced ultrasound, $\mathrm{CT}=$ computed tomography, $\mathrm{US}=$ ultrasound, $\mathrm{MRI}=$ magnetic resonance imaging .

whereby a small overlap exists due to misinterpretation in the initial imaging, which was maintained for statistical reasons.

The indication for CEUS was given in the case of the presence of indeterminate renal lesions on initial imaging (CT: 307, US: 160, MRI: 32). In six outpatients the initial imaging modality could not be determined. Since CEUS requires a separate contrast agent application for each lesion, indication for a CEUS examination was given only for patients with a maximum number of two lesions for both kidneys. Patients with more lesions were examined with complementary sectional imaging (CT or MRI), depending on the initial imaging (CT, MRI, US), unless there was a contraindication for the corresponding examination. In the 54 patients with examination of both kidneys, there was only one lesion per kidney. Only one patient underwent a CEUS examination for three lesions of the right kidney.

The contrast agent used in this study was BR1 (SonoVue, Bracco ALTANA Pharma, Constance, Germany). All CEUS examinations were performed on an Acuson Sequoia 512 (Siemens Ultrasound, Mountainview, CA, USA) or a Toshiba Aplio 500 (Toshiba Europe, Zoetermeer, $\mathrm{NL}$ ) using a $3.5 \mathrm{MHz}$ ultrasound probe (4C1). The examination protocol included an initial B-mode ultrasound scan to get an overview of the lesion and the surrounding renal parenchyma and Doppler imaging to assess vascularization. This was followed by cubital i. v. application of $1.2 \mathrm{ml}$ SonoVue as a bolus injection and post-injection of $10 \mathrm{ml}$ of a $0.9 \% \mathrm{NaCl}$ solution. Contrast-specific imaging techniques with a low mechanical index ("low 
Ml"<0.3) were used over a period of approximately 3 minutes. If the first injection did not provide sufficient diagnostic information, a second injection was administered, and the study was repeated. For digital documentation, short video clips of the cortical phase, the parenchymal/nephrogenic phase, and the late phase (>120 s post-injection) of each examination were recorded. All image data were stored in the local picture archiving and documentation system.
Atypical kidney configurations, e. g., prominent dromedary hump, were interpreted as pseudolesions. Pyelonephritic lesions, e. g., abscesses or perfusion deficits were interpreted as inflammatory lesions, and parenchymal retractions as ischemic lesions. All lesions were divided into cystic and solid lesions. The cystic lesions were classified according to Bosniak ( $\triangleright$ Figs. 3-6). Solid lesions were interpreted as potentially malignant if they were not unambiguously identified as pseudo-, inflammatory, or ischemic lesions.
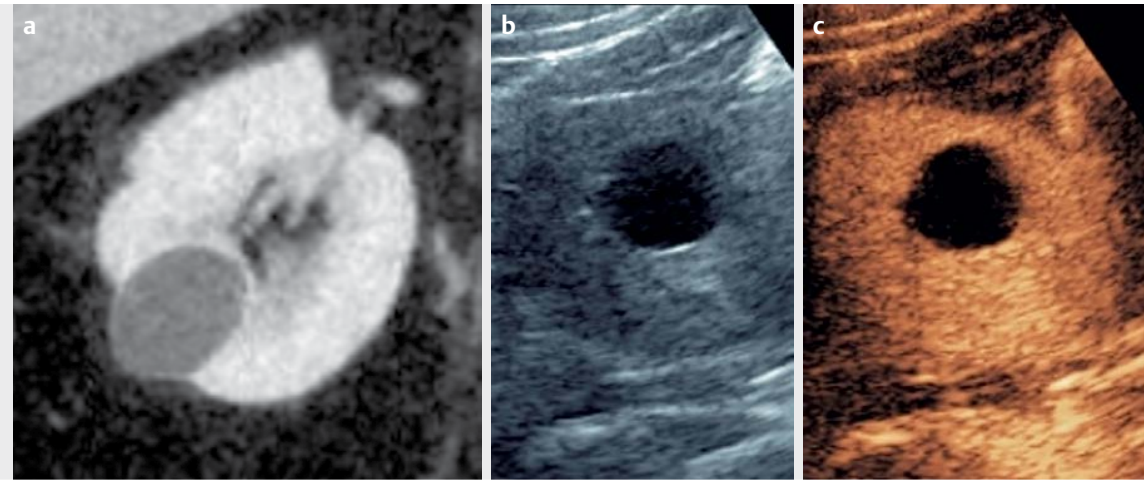

- Fig. 3 Uncomplicated cyst; Bosniak I. a Hypodense, smoothly bordered, and thin-walled lesion with a diameter of $2.5 \mathrm{~cm}$ and water equivalent density values on contrast-enhanced CT. $\mathbf{b}$ On the native image an interface echo and dorsal acoustic enhancement are visible. c On CEUS the lesion shows a tender wall, no septation, and no enhancement, consistent with an uncomplicated benign renal cyst.
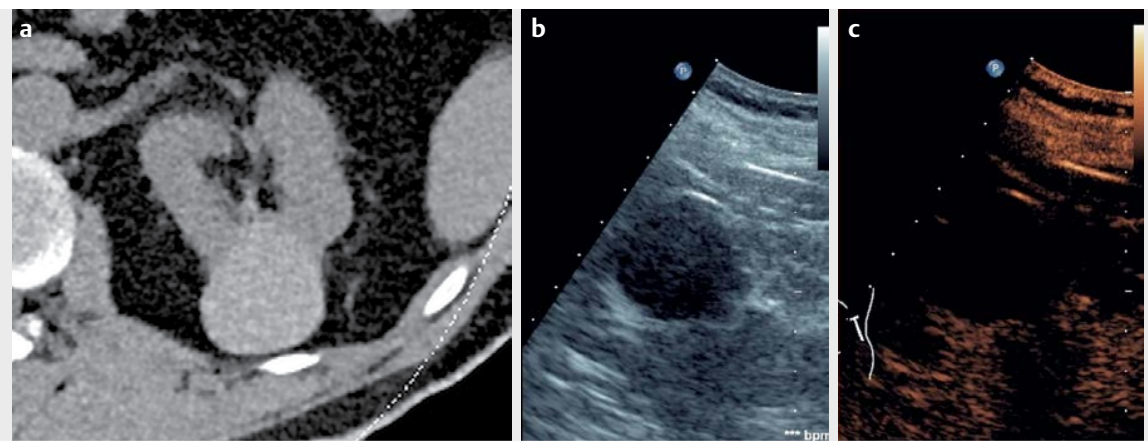

- Fig. 4 Minimally complicated homogeneous hyperdense cyst; Bosniak II a Homogeneous hyperdense lesion in the pars intermedia of the left kidney on native CT with density values of 75 Hounsfield units. b On the native image, no septae or solid parts are detected. c On CEUS there is no contrast enhancement, consistent with an exsanguinated/protein-rich cyst.
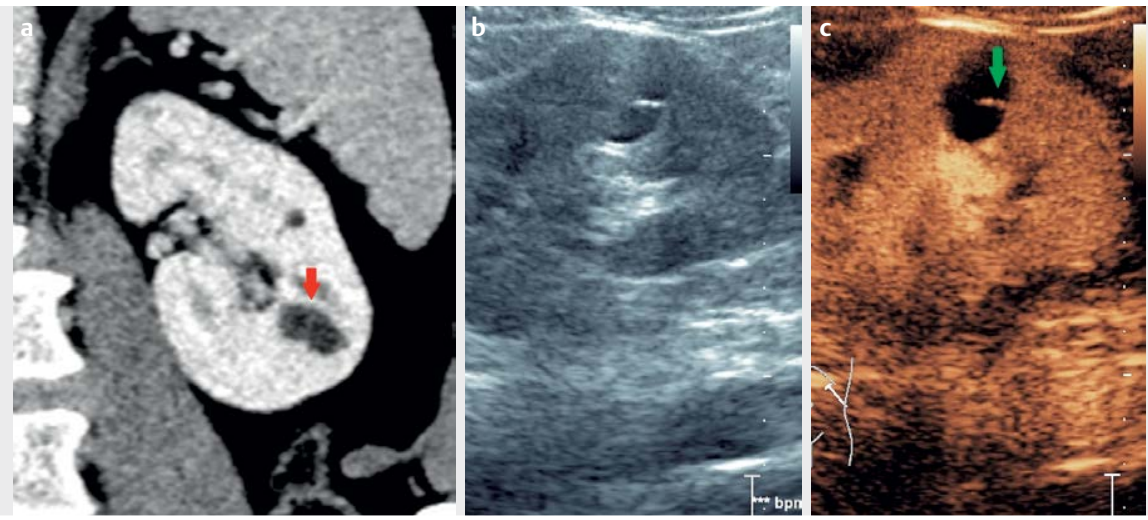

- Fig. 5 Complicated, septated cyst; Bosniak IIF. a $2.5 \mathrm{~cm}$ hypodense lesion at the lower pole of the left kidney with fine septation on contrast-enhanced CT (red arrow). b and $\mathbf{c}$ Native and contrast-enhanced ultrasound show a $1.5 \mathrm{~mm}$ septum with discrete contrast enhancement (green arrow). However, there is no nodular component. 

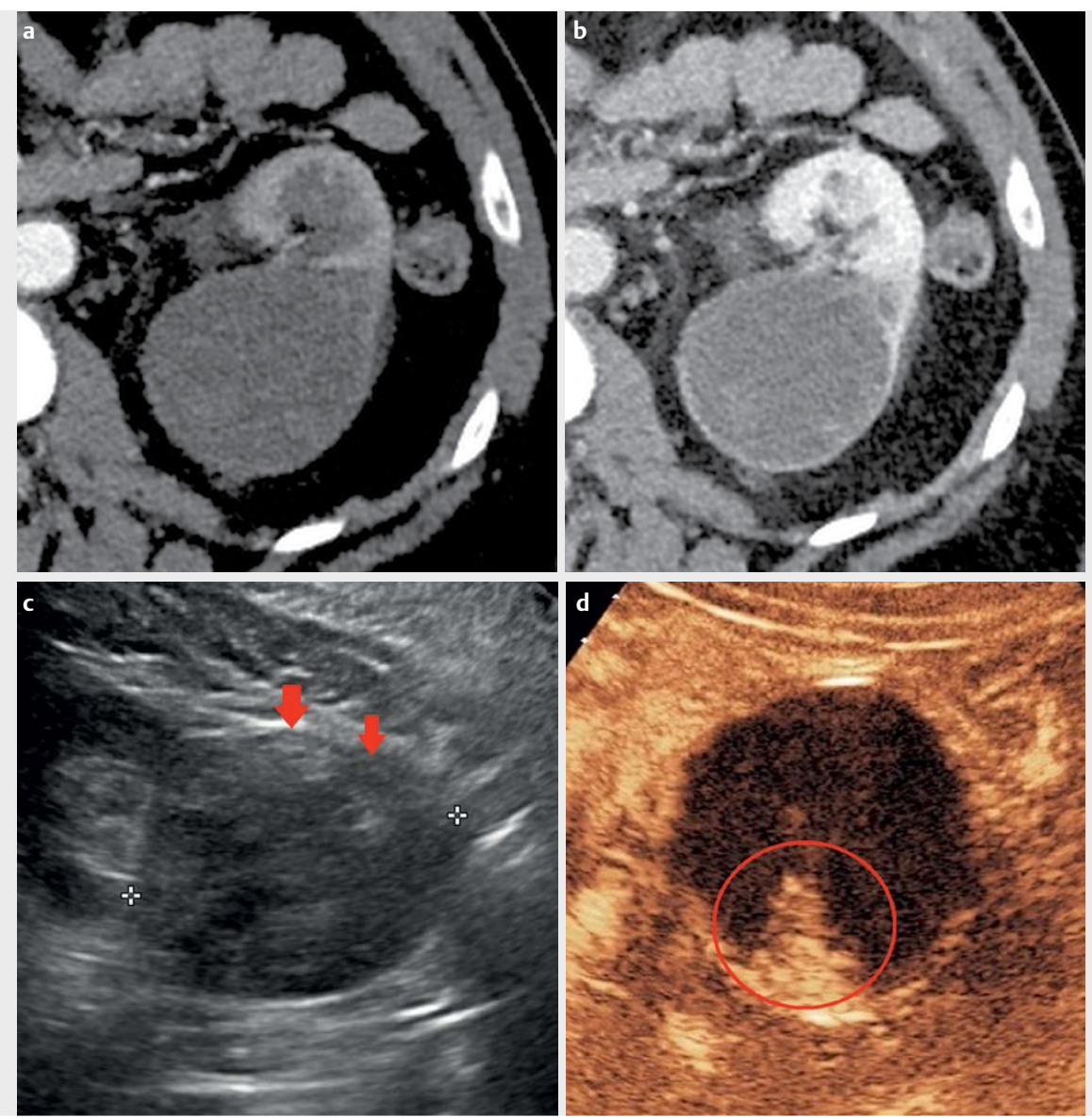

- Fig. 6 Highly suspicious lesion; Bosniak IV. a $6 \mathrm{~cm}$ hypodense lesion in the pars intermedia of the left kidney with a density between 20 and 30 Hounsfield units on contrast-enhanced CT (arterial phase). $\mathbf{b}$ In the portal venous phase, the lesion shows density values up to 40 Hounsfield units, but with enhancement of the septae. $\mathbf{c}$ On the B-mode image, low echoes with solid, echo-rich parts are displayed (arrows). $\mathbf{d}$ CEUS shows strong partial enhancement (circle) extending to the center, matching the vessels at the edges of the lesion. The lesion was classified as a partially cystic, partially solid tumor (Bosniak IV). Histologically it was a papillary renal cell carcinoma.

The lesions that were classified as benign on CEUS were classified as "definitely benign" if the initial imaging also did not reveal any malignancy criteria. Histologically confirmed lesions could be clearly divided into malignant and benign lesions. Lesions that were not clearly malignant (Bosniak IIF) were further followed up. These lesions were assigned to a category depending on imaging findings during the course of these follow-ups. To definitely classify a lesion as malignant, either histological clarification was required or at least two different imaging modalities with strong malignancy criteria (e. g., clear interval growth, invasion of surrounding structures, other tumor manifestations, etc.).

The sensitivity, specificity, positive and negative predictive value as well as positive and negative likelihood ratios (PLR/NLR) were calculated.

\section{Results}

In total, there were 437 benign and 54 malignant lesions. Of the benign lesions, 31 were inflammatory and 5 were ischemic. Cystic lesions with Bosniak classification were present in 282 cases, solid lesions in 209 cases. Most of the solid lesions were pseudolesions $(n=98)$, followed by renal cell carcinomas $(n=42)$, inflammatory lesions $(n=29)$, and angiomyolipomas $(n=24)$. All other lesions were significantly less frequent $(n=1-4)$.

Of the 491 statistically evaluated lesions, 417 were correctly classified as benign, 53 correctly as malignant, 20 falsely as malignant, and only one lesion was falsely classified as benign. Accordingly, the sensitivity for malignant lesions was 0.981 with a specificity of 0.954 , PPV of 0.726 , and NPV of 0.998 . For all evaluated lesions taken together, the PLR was 21.4 and the NLR 0.024.

\section{Cystic lesions}

A total of 301 lesions were classified as cystic, 274 of which were benign and 27 malignant (with 17 being renal cell carcinomas) in the conclusive diagnosis. There was one pseudolesion, two adenomas, and two angiomyolipomas ( $\bullet$ Fig. 7). No inflammatory or ischemic lesions were found in this group.

Of all cystic lesions, 262 were correctly classified as benign and 27 were correctly classified as malignant. Only 12 lesions were falsely classified as malignant, and none as falsely benign. Accord- 


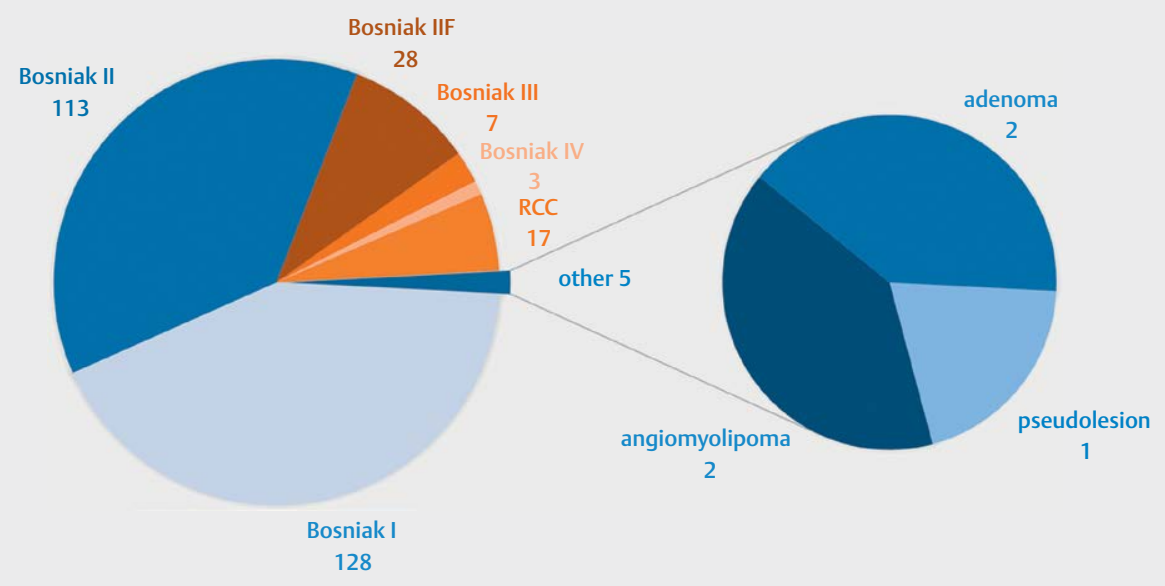

- Fig. 7 Final classification of the cystic lesions. A total of 301 lesions were classified as cystic, 274 as benign, and 27 as malignant. The majority of the benign lesions were uncomplicated renal cysts Bosniak types I and II ( 241 of $274,89 \%$ ) which do not require further evaluation. RCC was histologically diagnosed in 17 of the cystic lesions (6\%). (orange/red = (potentially) malignant, blue = benign).

ingly, the sensitivity for malignancy was 1,000 for cystic lesions with a specificity of 0.956 , a PPV of 0.692 , and an NPV of $1,000$. The PLR was 22.7 and the NLR was 0.01 .

Regarding the correct classification of the benign lesions according to Bosniak, 123 lesions were classified as type I, 96 as type II, and 26 type as IIF cysts, i. e., a total of 245 of 274 (89\%) were correctly classified as benign.

\section{Solid Lesions}

A total of 215 lesions were classified as solid ( $\mathbf{F i g . 8}$ ), 171 of which were benign and 44 malignant (with 42 being renal cell carcinomas). Frequent types of lesions were pseudo-lesions (98), inflammatory lesions (29), or angiomyolipomas (24). All other types were represented significantly less frequently $(n<6)$ ( $\mathbf{F i g}$. 9).

Of the solid lesions, 155 were correctly classified as benign and 43 correctly as malignant, 16 lesions were falsely classified as malignant and only one lesion was falsely classified as benign. Accordingly, the sensitivity for malignancy was 0.977 for the solid lesions, the specificity was 0.906 , the PPV was 0.729 , and the NPV was 0.994 . For the solid lesions, the PLR was 10.4 and the NLR was 0.025 .

\section{Histologically confirmed lesions}

The group of histologically confirmed lesions comprises 49 pre-selected, potentially malignant lesions. Accordingly, there were 35 (71\%) malignant lesions and 14 (29\%) benign lesions. The malignant lesions included 34 renal cell carcinomas and one metastasis of a pancreatic carcinoma. The benign lesions included 5 cystic lesions, 3 angiomyolipomas, 3 adenomas, 2 oncocytomas, and 1 leiomyoma ( $\triangleright$ Fig. 10).

Of the 25 biopsied solid lesions, 18 (72\%) were malignant, while this was the case in 12 (86\%) of the 14 biopsied Bosniak IV cysts and 10 (50\%) of the 20 biopsied Bosniak III cysts ( $\bullet$ Fig. 11). These results are comparable to findings in the literature $[19,20]$.

The calculated values for sensitivity, specificity, PPV, and NPV in the histologically confirmed lesions were $0.971,0.071,0.723$, and
0.500 , respectively. The PLR was 1.05 and the NLR was 0.4. However, the statistical indicators are of limited use due to the strongly introduced bias to include malignant lesions.

\section{Summary of statistics}

For clarity, all statistical indicators are summarized in $>$ Table 1. For imaging modalities the values are almost all in a very high range with regard to sensitivity, specificity, NPV, PLR, and NLR, and in an acceptable range with regard to PPV. The relatively low specificity, NPV, and PLR values for the histologically confirmed lesions are most likely explained by the small group size and strong bias.

\section{Discussion}

The aim of the present study was to show that contrast-enhanced ultrasound is suitable for the characterization of initially unclear kidney lesions in the everyday clinical setting.

As expected, cystic lesions accounted for the largest proportion. In our study 241 of 301 (80\%) cystic lesions fell into the Bosniak I or Il category, which required no further investigation. Complicated cysts classified as type IIF, III, or IV were more important indications for CEUS. Compared to the meta-analysis of Lan et al. [21], where the pooled sensitivity of CEUS of malignant cystic lesions was 0.95 and the pooled specificity was 0.76 , our results are significantly better. Accordingly, the PLR and NLR with 22.7 versus 4.39 and 0.01 versus 0.10 were also better. Lan et al. also evaluated contrast-enhanced $C T$, with a sensitivity of 0.90 , specificity of 0.79 , PLR of 5.00, and NLR of 0.15 . Thus, the results with contrast-enhanced CT within the Lan et al. study was more similar to the values obtained with CEUS in our study. In particular, the advantages of CEUS over CT are the high-resolution imaging of fine septations and the real-time imaging of contrast agent dynamics. In many cases, this allows reliable classification of cystic kidney lesions [22]. CEUS is particularly well suited for monitoring the progression of complex cystic lesions, especially in the case of Bosniak IIF cysts, since long-term computed tomographic monitoring of the pro- 

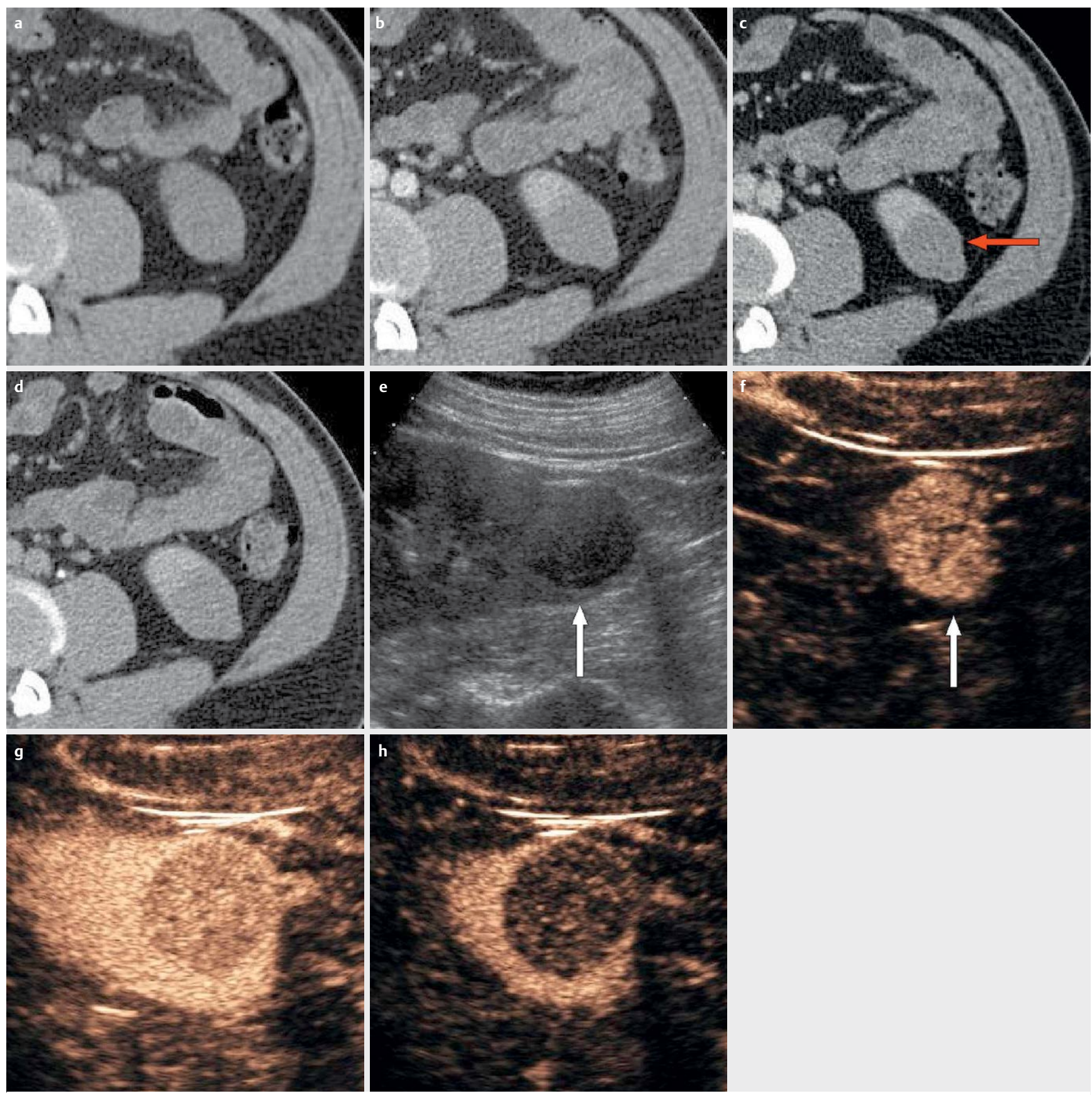

- Fig. 8 Solid malignant lesion, histologically papillary renal cell carcinoma. a-d Even on multiphase contrast-enhanced CT, no clear contrast agent uptake (i.e., $\mathrm{HU}>10$ ) could be detected. The density values varied between a native: $29 \mathrm{HU}$, b arterial: $35 \mathrm{HU}$, c portal venous: $38 \mathrm{HU}$, and d late: 36 HU. e Native ultrasound shows a hypoechogenic (arrow) lesion. f-h On CEUS, there is rapid homogeneous arterial contrast enhancement (arrow) as well as $\mathrm{g}$ venous and $\mathrm{h}$ late washout. $\mathrm{HU}=$ Hounsfield units.

gression of the disease over many years means a considerable cumulative radiation dose for patients, which is especially relevant in younger patients. Indeed, in our study 185 of 449 patients ( $41 \%)$ were under the age of 60 years. Additionally, renal abscesses or other inflammatory changes can also be easily evaluated.

As for solid lesions, CEUS can greatly help assess atypical renal configurations that mimic malignancy. A distinction between cystic and solid lesions is always possible. As far as further differentiation of solid lesions is concerned, CEUS, like all other imaging mo- dalities, is not helpful since there are no reliable criteria to distinguish solid benign from solid malignant lesions. Additionally, about $5 \%$ of solid renal tumors show iso-enhancement on CEUS [22].

Limitations of CEUS were similar to those of conventional ultrasound, including difficult visibility due to lesion location, obese patients, or intestinal gas superimposition, and steep learning curve for beginners.

Limitations of the study include retrospective design, multiple radiologists performing the CEUS examinations, histological con- 


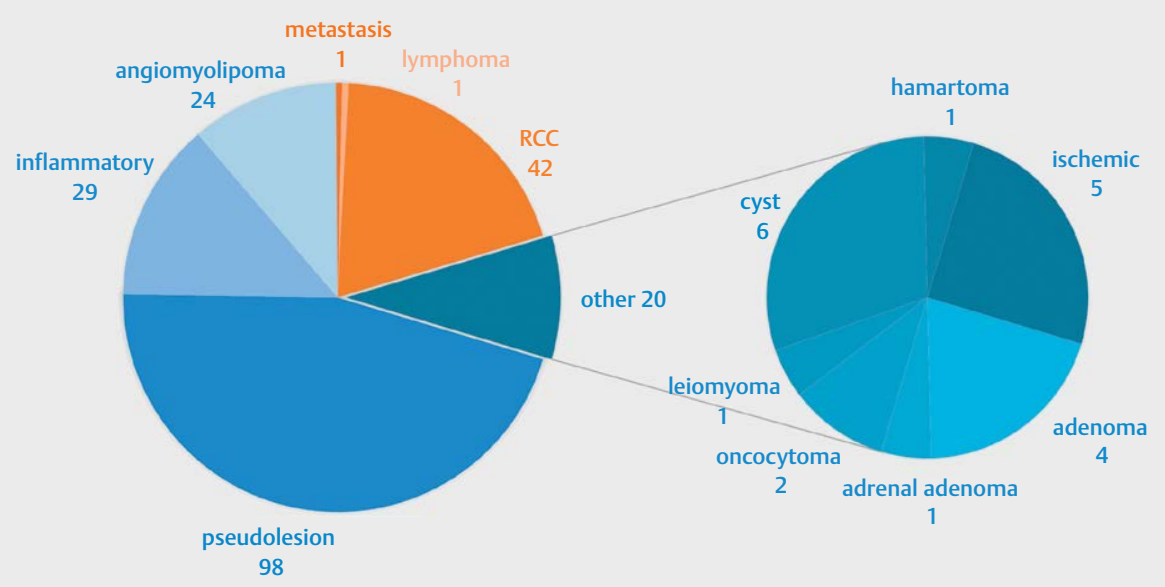

- Fig. 9 Final classification of solid lesions. A total of 215 lesions were classified as solid, with 171 of them classified as benign and 44 as malignant. The benign lesions included 98 pseudotumors, 29 inflammatory lesions, and 24 angiomyolipomas. The remaining lesions were significantly less frequent (<6). 42 of the malignant lesions were RCCs (95\%) (red = malignant, blue = benign). RCC = renal cell carcinoma.

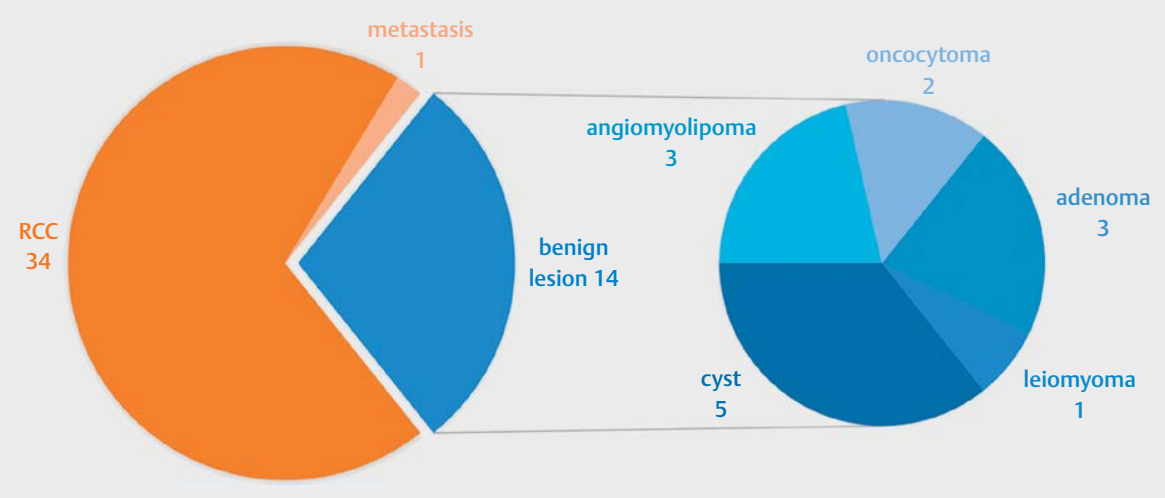

- Fig. 10 Histological findings of the biopsied lesions. A total of 49 of the 491 statistically evaluated lesions were biopsied. The histological findings revealed 35 malignant lesions, 34 of which were RCCs and one was a metastasis. A total of 14 benign lesions were found, whereby the two oncocytomas were evaluated as benign in this study (red = malignant, blue = benign).

firmation in only relatively low number of cases (10\%), which is due to the explicitly mentioned clinical setting and was also not feasible for ethical reasons. In particular, the classification as "definitely benign" and the associated discontinuation of further follow-up examinations and the omission of histological clarification may have led to a bias in the results.

However, the exclusively clinical setting in which these lesions were evaluated can also be regarded as a strength of the study, since it shows results in the everyday setting rather than confined study environment. This allows us to determine whether CEUS produces sufficiently useful results even under less controlled conditions.

To conclude, CEUS is a suitable tool for the evaluation of cystic and solid renal lesions that have been detected by other imaging modalities in the everyday clinical setting and whose status as malignant or benign is unclear. 


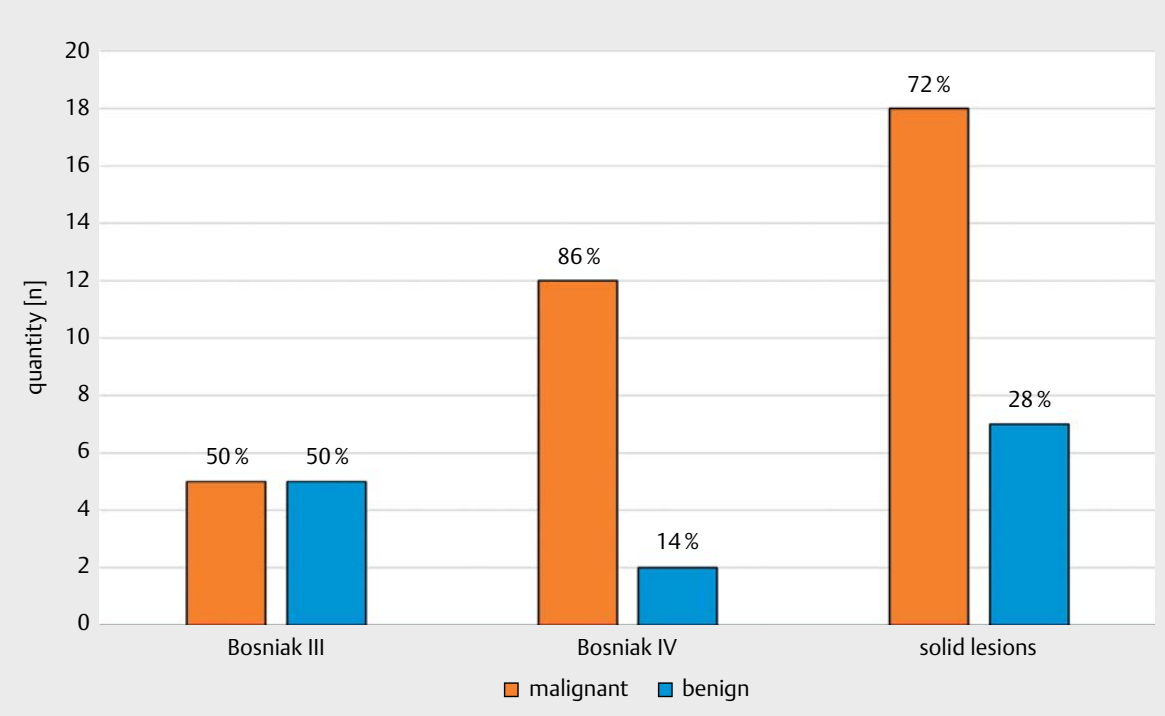

- Fig. 11 Malignant and benign histology of suspicious lesions. Subdivision of the biopsied Bosniak III/IV lesions and the solid lesions according to classification on CEUS and final histological findings. Out of 10 potentially malignant Bosniak III cysts, 5 were malignant. Of the Bosniak IV cysts clearly classifiable as malignant, 12 of 14 cysts were malignant and 18 of the 25 biopsied solid lesions were malignant. Orange $=$ malignant, blue $=$ benign.

- Table 1 Statistical indicators of all lesions and subgroups.

\begin{tabular}{|l|l|l|l|l|l|l|}
\hline Lesions & Sens & Spec & PPV & NPV & PLR \\
\hline All & 0.981 & 0.954 & 0.726 & 0.998 & 21.4 & 0.024 \\
\hline Cystic & 1.000 & 0.956 & 0.692 & 1.000 & 22.7 & 0.010 \\
\hline Solid & 0.977 & 0.906 & 0.729 & 0.994 & 10.4 & 0.025 \\
\hline Histologically confirmed & 0.971 & 0.071 & 0.723 & 0.500 & 1.05 & 0.400 \\
\hline
\end{tabular}

Sens = sensitivity, $\mathrm{Spec}=$ specificity, PPV = positive predictive value, NPV = negative predictive value, PLR = positive likelihood ratio, NLR = negative likelihood ratio.

\section{Conflict of Interest}

The authors declare that they have no conflict of interest.

\section{References}

[1] Mazziotti S, Cicero G, D’Angelo T et al. Imaging and Management of Incidental Renal Lesions. Biomed Res Int 2017; 2017: 1854027

[2] Bosniak MA. The current radiological approach to renal cysts. Radiology 1986; 158: 1-10

[3] Israel GM, Bosniak MA. An update of the Bosniak renal cyst classification system. Urology 2005; 66: 484-488

[4] Silverman SG, Pedrosa I, Ellis JH et al. Bosniak Classification of Cystic Renal Masses, Version 2019: An Update Proposal and Needs Assessment. Radiology 2019; 292: 475-488

[5] Huber J, Hallscheidt P, Wagener $\mathrm{N}$ et al. Raumforderungen der Niere: CT vs. MRT. Zwei fast gleichwertige Alternativen mit feinen Unterschieden [Tumours of the Kidney: CT vs. MRI. Nearly equal alternatives with minor differences]. Urologe A 2010; 49: 345-350. German
[6] Smith-Bindman R, Lipson J, Marcus R et al. Radiation dose associated with common computed tomography examinations and the associated lifetime attributable risk of cancer. Arch Intern Med 2009; 169: 2078-2086

[7] Di Vece F, Tombesi P, Ermili F et al. Management of incidental renal masses: Time to consider contrast-enhanced ultrasonography. Ultrasound 2016; 24: 34-40

[8] Claudon M, Dietrich CF, Choi Bl et al. Guidelines and good clinical practice recommendations for Contrast Enhanced Ultrasound (CEUS) in the liver - update 2012: A WFUMB-EFSUMB initiative in cooperation with representatives of AFSUMB, AIUM, ASUM, FLAUS and ICUS. Ultrasound Med Biol 2013; 39: 187-210

[9] Piscaglia F, Bolondi L. Italian Society for Ultrasound in Medicine and Biology (SIUMB) Study Group on Ultrasound Contrast Agents. The safety of Sonovue in abdominal applications: Retrospective analysis of 23188 investigations. Ultrasound Med Biol 2006; 32: 1369-1375

[10] Ascenti G, Gaeta M, Magno C et al. Contrast-enhanced second-harmonic sonography in the detection of pseudocapsule in renal cell carcinoma. AJR Am J Roentgenol 2004; 182: 1525-1530

[11] Park BK, Kim B, Kim SH et al. Assessment of cystic renal masses based on Bosniak classification: comparison of CT and contrast-enhanced US. Eur J Radiol 2007; 61: 310-314 
[12] Tamai H, Takiguchi Y, Oka M et al. Contrast-enhanced ultrasonography in the diagnosis of solid renal tumors. J Ultrasound Med 2005; 24: 1635-1640

[13] Ascenti G, Mazziotti S, Zimbaro G et al. Complex cystic renal masses: characterization with contrast-enhanced US. Radiology 2007; 243: 158-165

[14] Clevert DA, Minaifar N, Weckbach S et al. Multislice computed tomography versus contrast-enhanced ultrasound in evaluation of complex cystic renal masses using the Bosniak classification system. Clin Hemorheol Microcirc 2008; 39: 171-178

[15] Piscaglia F, Nolsøe C, Dietrich CF et al. The EFSUMB Guidelines and Recommendations on the Clinical Practice of Contrast Enhanced Ultrasound (CEUS): update 2011 on non-hepatic applications. Ultraschall in Med 2012; 33: 33-59

[16] Mazziotti S, Zimbaro F, Pandolfo A et al. Usefulness of contrast-enhanced ultrasonography in the diagnosis of renal pseudotumors. Abdom Imaging 2010; 35: 241-245

[17] Correas JM, Claudon M, Tranquart F et al. The kidney: imaging with microbubble contrast agents. Ultrasound Q 2006; 22: 53-66
[18] Sidhu PS, Cantisani V, Dietrich CF et al. The EFSUMB Guidelines and Recommendations for the Clinical Practice of Contrast-Enhanced Ultrasound (CEUS) in Non-Hepatic Applications: Update 2017 (Long Version). Ultraschall in Med 2018; 39: e2-e44

[19] Harvey C], Alsafi A, Kuzmich S et al. Role of US Contrast Agents in the Assessment of Indeterminate Solid and Cystic Lesions in Native and Transplant Kidneys. Radiographics 2015; 35: 1419-1430

[20] Bertolotto M, Cicero C, Perrone R et al. Renal Masses With Equivocal Enhancement at CT: Characterization With Contrast-Enhanced Ultrasound. AJR Am J Roentgenol 2015; 204: W557-W565

[21] Lan D, Qu HC, Li N et al. The Value of Contrast-Enhanced Ultrasonography and Contrast-Enhanced CT in the Diagnosis of Malignant Renal Cystic Lesions: A Meta-Analysis. PLoS One 2016; 11: e0155857. doi:10.1371/journal.pone.0155857

[22] Bertolotto M, Cicero C, Catalano O et al. Solid Renal Tumors Isoenhancing to Kidneys on Contrast-Enhanced Sonography: Differentiation From Pseudomasses. J Ultrasound Med 2018; 37 : 233-242 